\title{
On the similarity methods in fracture mechanics
}

\author{
Ya-Pu Zhao
}

\begin{abstract}
This paper presents a brief review about the application of similarity methods to fracture mechanics. This includes some dimensionless numbers and some scaling laws in fracture mechanics, discrete scale invariance (DSI) and log-periodic corrections to scaling, the analogy between multiple fracture of solids and fully developed turbulence, and the property of self-organized criticality of some fracture process. An example is given to show the application of similarity method for the solution of a stationary semi-infinite mode III crack subjected to step dynamic loading. As a self-similar problem, the governing equation can be reduced to Riemann's equation by a simple similarity transformation, and Riemann's equation can be reduced to Gauss' hypergeometric equation by a certain transformation, thus this problem is solved by using the hypergeometric series.

\section{Über die Verwendung von Ähnlichkeitsgrößen in der Bruchmechanik}

Zusammenfassung In diesem Artikel wird zunächst ein kurzer Einblick in die Verwendung von ÄhnlichkeitsgröBen in der Bruchmechanik gegeben. Hierzu werden einige dimensionslose Größen und Ählichkeitsgesetz der Bruchmechanik, diskrete Ähnlichkeitsinvarianten und logarithmisch-periodische Korrekturen, die Analogie zwischen Mehrfachbruch in Festkörpern und der ausgebildeten, turbulenten Strömung sowie die Eigenschaft der Selbstorganisation bei einigen Bruchvorgängen vorgestellt. Am Beispiel eines stationären Bruchvorgangs eines halbunendlichen Risses unter stufenartiger, dynamischer Belastung (Mode III) wird der Einsatz von

Ähnlichkeitsgrößen aufgezeigt. Die beschreibenden Gleichungen Können durch eine einfache Ähnlichkeitstransformation in die Riemannsche Gleichung überführt werden, die ihrerseits auf die Gaussche, hypergeometri-

Received: 10. June 1998

Ya-Pu Zhao

Laboratory for Nonlinear Mechanics of Continuous media (LNM), Institute of Mechanics, Chinese Academy of sciences, Beijing 100080, P. R. China

e-mail: yzhao@Inm. imech.ac.cn

This work is sponsored by the foundation of the director of the Institute of Mechanics, CAS, and the National Natural Science Foundation of China. The valuable suggestion and discussions given by Prof. Che-Min Cheng are gratefully acknowledged. sche Gleichung reduziert werden kann. Das Problem kann somit unter Verwendung von hypergeometrischen Reihen gelöst werden.

\section{List of symbols}

$a, b, c \quad$ parameters of the Gauss' hypergeometric equation

\section{$c_{s}$}

$\mathrm{Ca}$

$d_{T}$

E

$E(k)$

$f$

$f(\xi, \theta)$

$F(a, b ; c ; z)$

$G_{I C}$

$G(k)$

$H(t)$

i

\section{Ir}

$J_{I C}$

$k$

$K_{B j}$

\section{$K_{I C}$}

$\Delta K$

\section{$l, l_{*}$}

$m$

\section{$n$}

$N, N_{*}$

$P_{f}$

$r$

$s$

$s_{E}$

$s_{f}$

$t$

$u, v$

V

$z_{n}$

$Z$

$\alpha, \beta$

$x_{n}, \alpha_{n}^{\prime}$

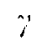

$\sqrt{\mu / \rho}$, shear stress wave speed

$\rho V^{2} / E$, Cauchy number

spacing of the second phase particles

Young's elastic modulus

power density spectrum

frequency

dimensionless function

hypergeometric series or hypergeometric function

energy release rate

power spectral density for various fracture surfaces

Heaviside unit step function

pure imaginary number

Irwin number

material J-integral fracture toughness

wavenumber

crack-branching

stress intensity factor

material fracture toughness

stress-intensity factor amplitude

characteristic dimension of a cracked

structure

defect size, mean defect size

exponent of the scaling law; $\frac{2 n+1}{2}$

in the example

integer

defect frequency, maximum frequency concentrated force

displacement from the crack-tip

Carpinteri's stress brittleness number

Carpinteri's energy brittleness number tension

time

dimensionless functions in (49)

crack speed

displacement in the $z$-direction

singular points, $n=1,2,3$

$E \sqrt{l} / K_{I C}$, dimensionless number

exponents

exponents belonging to $z_{n}, n=1,2.3$

surface energy 
$\Gamma$ (a) Gamma function 1

$\delta \quad$ crack opening displacement

$\delta(t) \quad$ delta function

$\Delta \quad$ displacement

$\epsilon \quad$ strain; radius of a very small hole at the crack-tip in (99)

$\frac{2 \zeta}{\zeta+1}$

angle

wavelength

$x_{n}-x_{n}^{\prime}$, exponent differences

elastic shear modulus

Poisson's ratio

$\frac{r}{c_{s} t}$

mass density

stress

ultimate stress of material

material yield stress

exponent

applied shear traction magnitude on the crack surfaces

Cherepanov's brittleness number

$\varkappa(x) \quad$ homogeneous function

Superscripts

$m$

model

$p$

prototype

\section{Introduction}

The concept of similarity has permitted essential and basic simplifications in the analysis of many engineering-physical phenomena. The application of similarity methods in fracture mechanics has a long established tradition. It is not very surprising that Leonardo da Vinci $(1452 \sim 1519)$ was already aware of the existence of model laws and mathematical relationships for the modes of behavior of geometrical similar structures. On the basis of similarity, he established a "comparative" theory for a very simple constructional shape (Fig. 1) [1]. Although man has built various, often highly complex, structures since ancient times, our knowledge about the strength and fracture of materials was long empirical and largely unstructured, transmitted from generation to generation as an art. The trail leading us to the scientific approach to problems of strength and fracture has been blazed by such pioneers as da Vinci and Galileo [2].

Leonardo da Vinci first conduct experiments to determine the load-bearing capacity of iron wires, he discovered the phenomenon called the scale effect, he stated that "Among cords of equal thickness the longest is the least strong". However, the achievements of da Vinci remained unknown by subsequent generations and therefore had no influence on the development of fracture mechanics, Galileo can rightfully be considered the founder of fracture me-
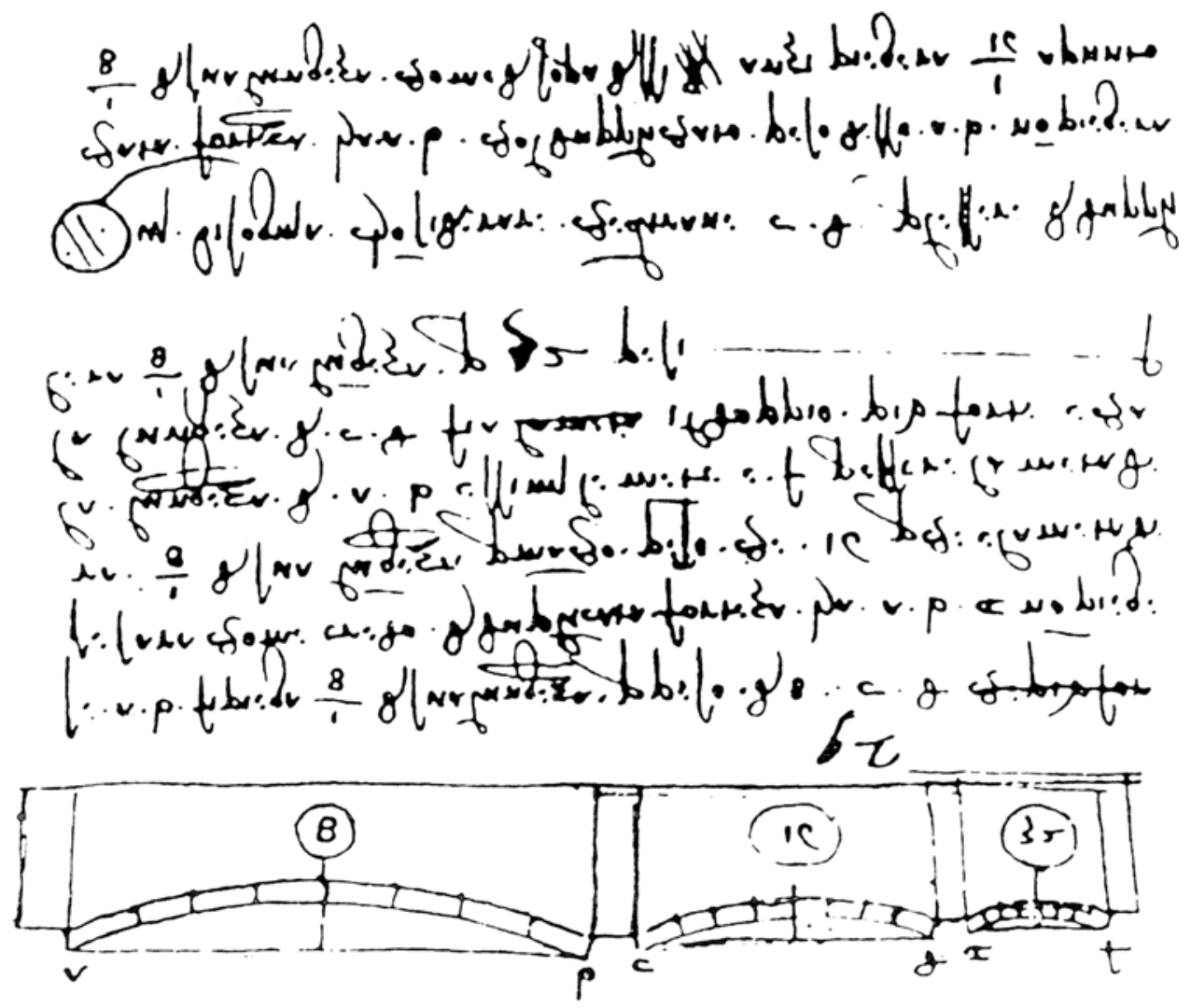

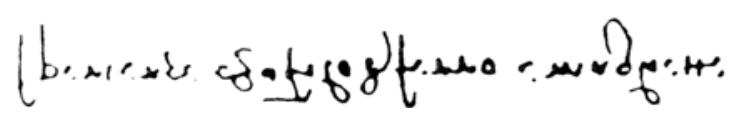

Fig. 1. Leonardo da Vinci study of similarity relationship 
chanics [2], due to his discovery that the fracture load of a bar in tension is directly proportional to its cross-sectional area and is independent of its length. Actually, the effect of the structure size on its nominal strength has long been a problem of central importance for both scientists and engineers. Galileo in 1638 pointed out "One cannot reason from the small to the large, because many mechanical devices succeed on a small scale that cannot exist in great size". He also pointed out "If the size of a body be diminished, the strength of that body is not diminished in the same proportion; indeed the smaller the body the greater its relative strength. Thus, a small dog could probably carry on his back two or three dogs of his own size, but I believe that a horse could not carry even one of his own size". It has experimentally been proven that a flea could carry on his back 2000 times his own weight.

About 500 years have past since da Vinci's famous experiments about the size effect of the cords. Fortunately, much progress has been achieved on the understanding about the scale effect of structure and material failure. Ref. [3] gave an extensive review about the scaling property of structural failure, interesting readers are referred there for details.

This article consists of two parts. In the first part, the application of similarity methods to fracture mechanics will be briefly reviewed. In the second part, an example will be given to show the application of similarity method to the solution of a stationary semi-infinite mode III crack problem subjected to step dynamic loading.

\section{2}

\section{Brief review of application of similarity methods to fracture mechanics}

\section{1}

\section{Some dimensionless numbers in fracture mechanics}

For the application of dimensional analysis to fracture mechanics, and particularly in setting up experiments, it is very important to select the dimensionless parameters correctly. As a matter of fact, dimensionless numbers have played a prominent role in fluid mechanics more than a hundred years ago. There should be as few parameters as possible in analyzing a practical problem, and they must reflect the fundamental effects in the most convenient way $[4,5]$, so it is important to derive the dimensionless products of variables that govern the fracture behavior of materials.

Cherepanov's brittleness number is defined by [2]

$\chi=\frac{E \gamma}{\sigma_{y}^{2} l}$,

where $E$ and $\sigma_{y}$ are, respectively, Young's elastic modulus and yield strength of material, $\gamma$ is the surface energy, and $l$ the characteristic length of the cracked body. For plane stress problem $K_{I C}^{2}=2 E_{\gamma}$, then the Cherepanov's brittleness number is

$\chi=\frac{1}{2} \frac{K_{I C}^{2}}{\sigma_{y}^{2} l}$

where $K_{I C}$ is the fracture toughness of the material. Since the size of the plastic zone near the crack tip is of the order $r_{p} \sim\left(K_{I C} / \sigma_{y}\right)^{2}$, then $\chi$ in (2) can be considered the order of the ratio of the plastic zone size to the characteristic length of the cracked body. [6]

Carpinteri's stress brittleness number is expressed by

$$
s=\frac{K_{I C}}{\sigma_{u} \sqrt{l}},
$$

where $\sigma_{u}$ is the ultimate stress of the material. If we substitute $\sigma_{y}$ for $\sigma_{u}$ in (3), then Carpinteri's stress brittleness number can be considered the order of the square root of the ratio of the crack-tip plastic zone size to the characteristic dimension of the structure [7]. Carpinteri's energy brittleness number for linear elastic material is defined by [8]

$s_{E}=\frac{G_{I C}}{\sigma_{u} l}$

where $G_{I C}$ is the energy release rate, for plane stress problems we have $K_{I C}^{2}=E G_{I C}$, then (4) is changed into

$s_{E}=\frac{K_{I C}^{2}}{E \sigma_{u} l}$.

If we substitute $\sigma_{y}$ for $\sigma_{u}$ in (5), since the order of crack opening displacement (COD) can be expressed by $\delta \sim K_{I C}^{2} / E \sigma_{y}$, then $s_{E}$ can be interpreted as the order of the ratio of COD to the characteristic dimension of the cracked structure. The energy brittleness number in the case of Ramberg-Osgood material is then given by

$s_{E}^{*}=\frac{J_{I C}}{\sigma_{y} l}$,

where $J_{I C}$ is the J-integral fracture toughness.

The Irwin number defined by Barenblatt is $[9,10]$

$\mathrm{Ir}=\frac{\sigma_{y} \sqrt{l}}{K_{I C}}$

The Irwin number can be interpreted as the order of the square of the ratio of the crack-tip plastic zone size to the characteristic dimension of the cracked structure. The relationship among Irwin number, Cherepanov's brittleness number and Carpinteri's stress brittleness number is

$\operatorname{Ir} \sqrt{2 \chi}=1, \operatorname{Ir}=\frac{1}{s}$.

It has been proved by [11], that Irwin number can be used to predict ductile-brittle fracture transition, this conclusion was reached by dimensional analysis.

Zhao suggested a dimensionless number as [7]

$Z=\frac{E \sqrt{l}}{K_{I C}}$,

where $l$ is the characteristic dimension of the material's internal constraints (microstructures, defects, etc.) or external constraints (load or displacement boundary conditions, size of the structure or of the crack). If displacement boundary condition is given, then the general functional relationship can be expressed by

$\Delta=f\left(l, E, K_{I C}, v\right)$ 
where $\Delta$ and $l$ are, respectively, the applied displacement boundary condition and the characteristic dimension of the cracked body, $v$ is the Poisson's ratio. By Buckingham's $\pi$ theorem, (10) becomes

$\frac{\Delta}{l}=f\left(\frac{E \sqrt{l}}{K_{I C}}, v\right)$.

It is obvious that $Z=E \sqrt{l} / K_{I C}$ is a similarity parameter at this case. Some examples will be given below to show the usage of this dimensionless number in various cases. If we use the following Hollomon formula for alloy

$\sigma=A \varepsilon^{n}$,

and the Krafft's model

$K_{I C}=E n \sqrt{2 \pi d_{T}}$,

where $d_{T}$ is the spacing of the second phase particles, and if it is chosen to be the characteristic dimension of the internal constraint of the material, then we have

$Z=\frac{E \sqrt{d_{T}}}{K_{I C}}=\frac{1}{n \sqrt{2 \pi}}$.

For stainless steel $n=0.45 \sim 0.55$, then the values of the dimensionless number in (14) are approximately

$Z=0.73 \sim 0.89 ;$ for brass $n=0.35 \sim 0.4$, then from (14) we have $Z=1.0 \sim 1.14$. In studying the fractal characteristics of the ceramic fracture surface, Mecholsky and Freimann suggested the following empirical formula [12]

$$
\left(\frac{K_{I C}}{E}\right)^{2}=a_{0} D^{*}
$$

where $D^{*}$ is the fractional part of the fractal dimension and represents the amount of tortuosity out of the plane, $a_{0}$ is a parameter having the units of length. (15) can be changed into the following form

$\frac{E \sqrt{a_{0}}}{K_{I C}}=\frac{1}{\sqrt{D^{*}}}$.

A similar empirical formula has also been suggested

$E \frac{\sqrt{b_{0}}}{K_{B j}}=1$,

where $K_{B j}$ is the crack-branching stress-intensity factor, $b_{0}$ is also a parameter with the units of length. In studying the influence of finite deformation on the stress and strain fields near the crack tip, Cherepanov suggested the following relationship [2]

$\Delta=\frac{K_{I}^{2}}{E^{2}} \lambda\left(v_{1}, v_{2}, \ldots\right)$,

where $\Delta$ is the characteristic length of the finite deformation zone near the crack tip, and $\lambda\left(v_{1}, v_{2}, \ldots\right)$ is a dimensionless function. Equation (18) can be recast into the following form $\frac{E \sqrt{\Delta}}{K_{I}}=\lambda^{1 / 2}\left(v_{1}, v_{2} \ldots\right)$.
Cauchy number $\mathrm{Ca}=\rho V^{2} / E$ is one of the important dimensionless numbers in impact dynamics. The physical meaning of this dimensionless number in crack dynamics is the square of the ratio of crack speed to the characteristic speed of the elastic stress wave, or the square of the Mach number [7]. This dimensionless number is also a basic similarity parameter for elastic impact dynamics.

\section{2}

\section{Some scaling laws in fracture mechanics}

The fracture phenomena with which we shall be concerned all exhibit scaling. In its simplest form, this just means that two measurable quantities depend upon each other in a power-law fashion. In fact, the hallmark of scale invariance is the existence of power laws. Let us first recall what is the concept of scale invariance. Scale invariance means reproducing itself on different time or space scales. More precisely, an observable $\Omega$ which depends on a "control" parameter $x$ is scale-invariant under the arbitrary change $x \rightarrow \lambda x$, if there is a number $\mu(\lambda)$ such that

$\Omega(x)=\mu \Omega(\lambda x)$,

(19) defines a homogeneous function. Its solution is simply a power law $\Omega(x)=C x^{z}$, with $\alpha=-\log \mu / \log \lambda$, which can be verified directly by insertion. Power laws are the hallmark of scale invariance as the ratio $\Omega(\lambda x) / \Omega(x)=\lambda^{x}$ does not depend on $x$, i.e., the relative value of the observable at two different scales only depend on the ratio of the two scales. This is the fundamental property that associates power laws to scale invariance, self-similarity and criticality [13].

A remarkable example of the scaling law related to fracture mechanics, is the Benbow (1960) conical crack formed when a punch of small diameter is penetrating under a load $P$ into a block of fused silica having a large length-scale size $D$, the scaling law is

$P \sim D^{\frac{3}{2}}$,

which was quite satisfactorily confirmed by experiments.

The loads acting on the cracked structure are either forces, tensions (forces distributed along a line), or stresses depending on the way in which they are applied. Thus, the problem consists of determining the values of the force $P_{f}$, tension $s_{f}$, or stress $\sigma_{f}$ corresponding to the fracture of a structure on a geometrically similar model. These quantities are determined by a characteristic length of the structure $l$, by the fracture toughness $K_{I C}$, and by the dimensionless Poisson's ratio $v$. The general functional relationship is

$P_{f}, s_{f}, \sigma_{f}=g\left(l, K_{I C}, v\right)$.

when the loads (not displacements) are given, the Young's modulus does not enter the set of governing parameters [9, 10]. By Buckingham's $\pi$ theorem, we have

$\frac{P_{f}}{K_{I C} l^{\frac{3}{2}}}, \frac{s_{f}}{K_{I C} l^{\frac{1}{2}}}, \frac{\sigma_{f}}{K_{I C} l^{-\frac{1}{2}}}=g^{\prime}(v)$.

In the case of brittle fracture, equation (22) means that the only similarity parameter is the Poisson's ratio, so the similarity rules have the simple form 


$$
\begin{aligned}
\frac{P_{f}^{P}}{P_{f}^{m}} & =\frac{K_{I C}^{P}}{K_{I C}^{m}}\left(\frac{l^{P}}{l^{m}}\right)^{\frac{3}{2}}, \frac{s_{f}^{p}}{s_{f}^{m}} \\
& =\frac{K_{I C}^{p}}{K_{I C}^{m}}\left(\frac{l^{P}}{l^{m}}\right)^{\frac{1}{2}}, \frac{\sigma_{f}^{p}}{\sigma_{f}^{m}}=\frac{K_{I C}^{p}}{K_{I C}^{m}}\left(\frac{l^{p}}{l^{m}}\right)^{-\frac{1}{2}},
\end{aligned}
$$

by index $m$ we denote the model, by $p$ the prototype. If $K_{I C}^{P}=K_{I C}^{m}$, then from (23) we have

$\frac{\sigma_{f}^{p}}{\sigma_{f}^{m}}=\left(\frac{l^{p}}{l^{m}}\right)^{-\frac{1}{2}}$

which means that the intensity of the stress at corresponding points varies inversely as the square root of the scale $l^{p} / l^{m}$, this is called "scale effect".

If the plastic deformations incident to fracture are not confined to the narrow region around the crack, but occupy a significant part of the structure (this is called ductile fracture), the general functional relationship is given by

$P_{f}, s_{f}, \sigma_{f}=g\left(l, K_{I C}, \sigma_{y}, v\right)$,

where $\sigma_{y}$ is the yield stress. By Buckingham's $\pi$ theorem, we have

$\frac{P_{f}}{K_{I C^{\frac{3}{2}}}}, \frac{s_{f}}{K_{I C} l^{\frac{1}{2}}}, \frac{\sigma_{f}}{K_{I C} l^{-\frac{1}{2}}}=g^{\prime}($ Ir,$v)$.

In this case, we have two similarity parameters, the first one is the Poisson's ratio, and the second one is the Irwin number. If the similarity condition $v^{m}=v^{p}$ and $\operatorname{Ir}^{m}=\operatorname{Ir}^{p}$ are satisfied, the scaling of fracturing loads from model to prototype may be carried out using the same equations (23).

A classical result in the fatigue fracture is the scaling law by P. C. Paris and F. Erdogan (1963) for the crack propagation under multi-cycle fatigue

$\frac{\mathrm{d} a}{\mathrm{~d} n}=A(\Delta K)^{m}$,

where $\mathrm{d} a / \mathrm{d} n$ is the fatigue crack velocity per cycle averaged over the cycle and $\Delta K$ is the stress-intensity factor amplitude. The average velocity $\mathrm{d} a / \mathrm{d} n$ can depend on the following quantities: $\Delta K, l$ - the characteristic specimen size, $\sigma_{y}$ - yield stress, $f$ - frequency, $K_{I C}$ - fracture toughness, $t$ - time, $R=K_{\min } / K_{\max }$ - the asymmetry of loading. Dimensional analysis gives

$\frac{\mathrm{d} a}{\mathrm{~d} n}=\left(\frac{\Delta K}{\sigma_{y}}\right)^{2} \Phi\left(\frac{\Delta K}{K_{I C}}, R, \operatorname{Ir}, f \times t\right)$.

In fact, the parameter $\Delta K / K_{I C}$ is small, let us assume that the incomplete similarity takes place at the intermediate asymptotic stage of fatigue crack extension, then

$\Phi=\left(\frac{\Delta K}{K_{I C}}\right)^{\chi} \Phi^{\prime}(R, \mathrm{Ir})$

and $\alpha$, in principle, should also depend on the similarity parameter Ir. The values of $A$ and $m$ in (27) are

$m=2+\alpha, A=\left(\sigma_{y} K_{I C}\right)^{-(2+x)} \Phi^{\prime}(R, \mathrm{Ir})$.
Brown and Scholz [14] and Power et al. [15] computed the power spectral density, $G(k)$, for various rock fracture surfaces, natural joint surfaces in crystalline and sedimentary rocks, a bedding-plane surface, and frictional wear surfaces. Their results show that there is remarkable similarity among these surfaces. Profiles of these widely different surfaces yield power law of the form

$G(k)=B k^{-\chi}$,

where $k$ is the wavenumber related to the wavelength $\lambda$ of surface roughness according to $k=2 \pi / \lambda$. The exponent $\alpha$ has a fairly limited range (typically between 2 and 3 ). This power law form of the power spectrum indicates that fracture surface topography can be represented in terms of fractal geometry where the fractal dimension of the surface, $D$, is related to the power spectrum exponent as

$D=\frac{7-\alpha}{2}$.

For natural fracture surfaces, $D$ falls in the range of 2 to 3 , with small values representing smoother surfaces.

By using the critical point concept for rupture stress prediction from acoustic emissions, Anifrani et al. [16] suggested the following scaling law

$\frac{\mathrm{d} E}{\mathrm{~d} t}=E_{0}\left(p_{r}-p\right)^{-x}$,

where $\mathrm{d} E / \mathrm{d} t$ is the instantaneous $A E$ energy rate, $p$ and $p_{r}$ are the applied internal pressure and the rupture threshold, respectively, $\alpha$ is a so-called critical exponent, its range is $\alpha=1.5 \pm 0.2$. The nature of intermittency of the rupture would be involved in the log-periodic corrections to the leading scaling behavior

$\frac{\mathrm{d} E}{\mathrm{~d} t}=E_{0}\left(p_{r}-p\right)^{-x}\left\{1+C \cos \left[\phi+2 \pi \frac{\log \left(p_{r}-p\right)}{\log \hat{\lambda}}\right]\right\}$,

where $\log \lambda$ is the period, and $\phi$ is the phase.

If a great earthquake can be viewed as a critical point, precursors of earthquakes should follow characteristic scaling laws. In general, the scaling law should only be observable very near the critical point, in the so-called "critical region", and take the form [17]

$\frac{\mathrm{d} \epsilon}{\mathrm{d} t}=k\left|t_{f}-t\right|^{-x}$

where $\epsilon$ represents regional strain, $t_{f}$ is the time of failure. Integration of (35) yields

$\epsilon=A+B\left|t_{f}-t\right|^{m}$.

where $m=1-\chi$. Corrections of logarithmic periodicities lead to

$\epsilon(t)=A+B\left(t_{f}-t\right)^{m}\left\{1+C \cos \left[\psi+2 \pi \frac{\log \left(t_{f}-t\right)}{\log \lambda}\right]\right\}$. 


\section{3}

\section{Discrete scale invariance and log-periodic corrections to scaling}

Both equations (34) and (36) are two examples of logperiodic corrections to scaling. As a theoretical analysis, [18] predicted logarithmic oscillations in quasi-static crack growth. To our knowledge, Novikov has been the first to point in 1966 that structure factors in turbulence should contain log-periodic oscillations [19]. Loosely speaking, if an unstable eddy in turbulent flow typically breaks up into two or three smaller eddies, but not into 10 or 20 eddies, then one can suspect the existence of a preferable scale factor, hence the log-periodic oscillations.

It should be noted that the concept of discrete-scale invariance (DSI) leads to complex critical exponents (or dimensions), i.e. to the log-periodic corrections to scaling. Thus, the signature of DSI is the presence of power laws with complex exponents which manifests itself in data by log-periodic corrections to scaling [13].

From (19) we get the equation $1=\mu \lambda^{\alpha}$, but 1 is nothing but $\mathrm{e}^{\mathrm{i} 2 \pi n}$, where $n$ is an arbitrary integer, and $\mathrm{i}$ is the pure imaginary number. We then get

$\alpha=-\frac{\log \mu}{\log \lambda}+\mathrm{i} \frac{2 \pi n}{\log \lambda}$.

The special case $n=0$ gives the usual real power law solution corresponding to fully continuous scale invariance. In contrast, the more general complex solution corresponds to a possible DSI with the preferred scaling factor $\lambda$. The reason why equation (19) has solution in terms of complex exponents stems from the fact that a finite rescaling has been done by the finite factor $\lambda$. In critical phenomena presenting scale invariance, equation (19) corresponds to the linearization, close to the fixed point.

For the scaling property of the fully developed turbulence, Frisch [20] pointed out "An alternative way to improve the accuracy on scaling exponents (of turbulence) would be to fit the structure functions to power-laws with log-periodic corrections as was done by Sornette and Sammis [17] to improve the prediction of the time of occurrence of large earthquakes".

\section{4}

\section{Analogy between multiple fracture and developed turbulence}

In some conceptual aspect an analogy of multiple fracture with developed turbulence studies was found fruitful [ 9 , 10]. In the turbulence phenomenon we have the fluid instead of the deformable solid, the vortices instead of cracks and flows. The turbulence flow contains a cascade of interacting vortices of various length scales. We assume, therefore, the physical scheme of multiple fracture in the following way. There exists in a deformable solid a cascade of crack-like defects, pores, vacancies, dislocations etc. being in interaction.

Two independent processes exist simultaneously in the development of turbulence: the first one is the cascade process, in which large-scale forcing set up a cascade of energy transfers to smaller and smaller scales through nonlinearities of fluid motion. Eventually, at the smallest scales, viscosity caused the energy to be dissipated as heat; Another independent process is that small-scale vortices periodically pair so that larger fluid structures are created. The interactions of the defects could be, in principle, of two kinds: defects can either strengthen or weaken each other. Furthermore, the stress concentration near the tips of large defects stimulates the generation of new small defects. On the other side, the small defects stimulate the extension and coalescence of large ones.

Dislocation is the most important two-dimensional, or line, defect in solid, it is responsible for nearly all aspects of the plastic deformation of metals. The existence of a dislocationlike defect is necessary to explain the low values of yield stress observed in real crystals. Recent results indeed suggest that the fine scales of turbulent flow include a tangle of very intense and slender vortex filaments [20]. The filaments are actually tubes with an approximately circular cross-section, their diameter is of the order of the Kolmogorov dissipation scale. Moffatt et al. suggested that vortex filaments are the "sinews" of turbulence. Zhao [21] explained in detail the analogy of dislocation line in crystal with vortex filament in fluid. It is pointed out by [21] that the Burgers vector for screw dislocation corresponds to the circulation of a vortex filament, the shear strain round the screw dislocation to the flow velocity around the vortex filament.

In particular, there exists for a cascade the distribution curve of the defects size. Barenblatt and Botvina assumed the similarity principle: In multiple fracture the process of the development of the cascade of defects is statistically self-similar. Self-similarity means that when the damage accumulation is going on, the form of this curve remains fixed, only the maximum frequency $N_{*}$ and corresponding mean defect size $l_{*}$ are varying. Therefore the size distribution can be represented in the following universal form

$\frac{N}{N_{*}}=\Phi\left(\frac{l}{l_{*}}\right)$,

which is called Barenblatt-Botvina distribution. The distribution (38) is not a power law. The function $\Phi$ can be fitted by a curve [22]

$\ln \left(\frac{N}{N_{*}}\right)=-\ln ^{2}\left(\frac{l}{l_{*}}\right)$,

which was shown in Fig. 2. It should be noted [22] that not all self-similar and scaling properties of fracture can be described by methods of fractal geometry (e.g. the Barenblatt-Botvina distribution, log-periodicity).

\section{5}

\section{Self-organized criticality (SOC)}

It is interesting that many natural phenomena must be described by power law statistics. As pointed out previously, the hallmark of scale invariance is the existence of power laws. Correspondingly, an intense activity has developed in order to understand the origin of these ubiquitous power law tails. This has led in particular to the concept of "self-organized criticality" (SOC) [23], according to which certain dynamically driven spatially extended system evolve spontaneously towards a critical 


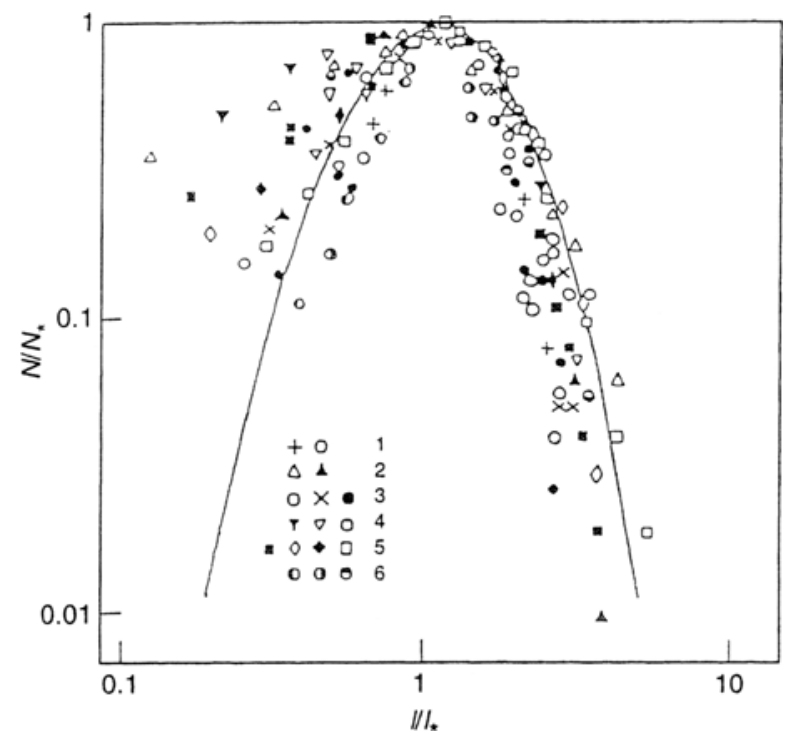

Fig. 2. The universal defect size distribution for various materials under various types of loading. 1. Polycrystalline copper, fatigue; 2. Brass, elastic tension; 3. Iron, creep; 4 . Steel 347, creep; 5 . Steel 304, creep; 6. Rocks

globally stationary dynamical state with no characteristic time or length scales.

Criticality refers to the state of a system which has scaleinvariant properties.

Several authors $[24,25]$ have argued that the earthquake phenomenology in geology is the signature of SOC. The Gutenberg-Richter law connecting the frequency distribution function with the energy release $E$ is

$\frac{\mathrm{d} N}{\mathrm{~d} E} \sim m^{-\tau}$,

with $1.25<\tau<1.5$, here $m$ is the earthquake size. Equation (40) is very similar to (27) since (27) can be simply rewritten as $\mathrm{d} n / \mathrm{d} a \sim(\Delta K)^{-m}$.

There are several kinds of scale invariance in the earthquake process, which are given in a power (scaling) law form in several empirical formulae [25]. For example, the size distribution of earthquakes in described by $N(s>S) \sim S^{-b}$, where $S$ is the area of the fracture zone of the earthquake, and the number of aftershocks decays as $n(t) \sim t^{-p}$.

In fact (31) is very similar with the Kolmogorov-Obukhov scaling law for fully developed turbulence

$E(k) \sim k^{-\beta}$.

where $E(k)$ is the power density spectrum, and $k$ is the wavenumber, $\beta=5 / 3$.

The scale invariance that determines these scaling exponents is a signature of SOC. Since long-range interactions are required to yield power laws, the experimental results suggest that both temporal and spatial interactions among the microcracks in the rock samples increased as the fracturing process progressed. Thereby, a self-similar fractal structure became self-organized during the fracture process [25].
3

\section{An example of application of self-similar method to dynamic fracture mechanics}

Self-similar method has long been used in wave propagation and crack dynamics. A novel method is given herein for a semi-infinite stationary anti-plane crack problem under step dynamic loading by using hypergeometric functions. This solution is also instructive for Modes I and II crack problems under the same dynamic loading.

3.1

\section{Formulation of the problem}

Consider a body of elastic material that contains a halfplane crack, but that is otherwise unbounded. The opposite faces of the crack are subjected to opposite, suddenly applied uniform traction in the $z$-direction, say $\sigma_{y z}(x, z, t)= \pm \tau^{*} H(t)$ on $y= \pm 0$, where $\tau^{*}$ is a constant traction magnitude and $H(t)$ is the Heaviside unit step function. Here we only consider the case of stationary crack, which means that the crack does not propagate under the action of dynamic loading. This problem is apparently a mode III (antiplane shear) one.

The wavefronts generated by the sudden application of the loading is shown in Fig. 3. For points near a crack face compared to the distance to the crack edge, the transient field consists only of a plane wave parallel to the crack face and traveling away from it at speed of shear stress wave $c_{s}=\sqrt{\mu / \rho}$, where $\mu$ and $\rho$ are elastic shear modulus and material mass density, respectively. As this wavefront passes a material point, the component of stress $\sigma_{z y}$ changes discontinuously from zero to $-\tau^{*}$, this can be expressed mathematically $\sigma_{z y}=-\tau^{*} H\left(c_{s} t-|y|\right)$.

The particle velocity changes discontinuously from zero to $\pm \tau^{*} / \rho c_{s}$ for $\pm y>0$, so the displacement field can be expressed by $w(x, y, t)= \pm \frac{t^{*}}{\mu}\left(y \mp c_{s} t\right) H\left(c_{s} t \pm y\right)$.

Near the crack edge, on the other hand, the field is more complex and some nonuniform field exists behind a cylindrical wavefront of radius $c_{s} t$ that is centered on the crack edge. This is the region in which the stress concentration develops.

The only nonzero component of displacement in the $z$ direction $w(x, y, t)$ satisfies the following wave equation [26]

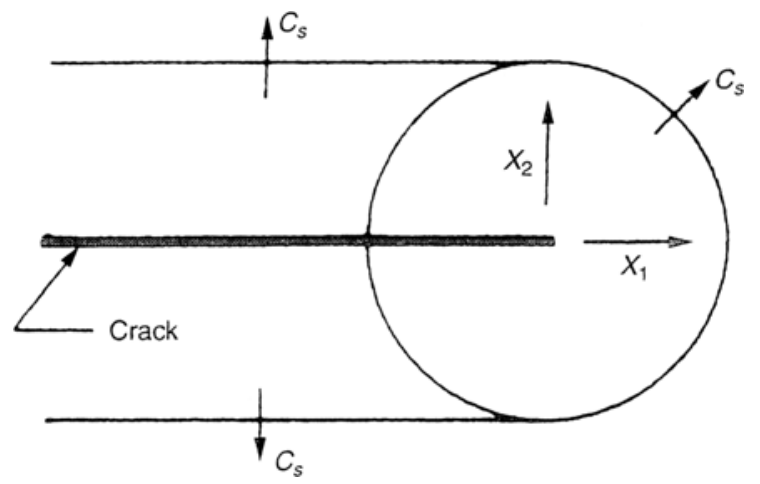

Fig. 3. Illustration of the wave propagation of a semi-infinite mode III crack problem under step dynamic loading 
$\frac{\partial^{2} w}{\partial x^{2}}+\frac{\partial^{2} w}{\partial y^{2}}=\frac{1}{c_{s}^{2}} \frac{\partial^{2} w}{\partial t^{2}}$

The solution of the wave equation (42) is subjected to the boundary and initial conditions

$$
\left\{\begin{array}{ll}
\mu \frac{\partial w\left(x, 0^{+}, t\right)}{\partial y}=-\tau^{*} H(t), & x \in(-\infty, 0) \\
w\left(x, 0^{+}, t\right)=0, & x \in(0, \infty) \\
\dot{w}(x, y, 0)=\frac{\partial w(x, y, 0)}{\partial t}=0 &
\end{array} .\right.
$$

264 The process being described involves neither a characteristic length nor a characteristic time with respect to which the independent variables may be scaled. Thus, this problem is self-similar. The similarity transformation are introduced as follows

$\frac{w(r, \theta, t)}{r}=f(\xi, \theta), \xi=\frac{r}{c_{s} t}$.

Due to the symmetry of the problem, only the upper half plane is necessary to be considered. By using similarity transformation (44), the displacement field for the particle near the upper face of the crack can be rewritten into

$$
\begin{aligned}
f(\xi, \theta)= & \frac{\tau^{*}}{\mu}\left(\sin \theta-\frac{1}{\xi}\right) H\left(\frac{1}{\xi}-\sin \theta\right), \\
& \xi \geq 1, \theta \in\left(\frac{\pi}{2}, \pi\right) .
\end{aligned}
$$

From (45) we have

$f(1, \theta)=\frac{\tau^{*}}{\mu}(\sin \theta-1), \theta \in\left(\frac{\pi}{2}, \pi\right)$,

and

$f(1, \theta)=0, \theta \in\left[0, \frac{\pi}{2}\right)$.

(46) and (47) are the boundary conditions that $f(\xi, \theta)$ should satisfy, these two equations are illustrated by Fig. 4 .

By substituting (44) into (42), we have

$$
\xi^{2}\left(1-\xi^{2}\right) \frac{\partial^{2} f}{\partial \xi^{2}}+\xi\left(3-2 \xi^{2}\right) \frac{\partial f}{\partial \xi}+f+\frac{\partial^{2} f}{\partial \theta^{2}}=0 .
$$

Separation of variables as

$f(\xi, \theta)=u(\xi) v(\theta)$,

gives

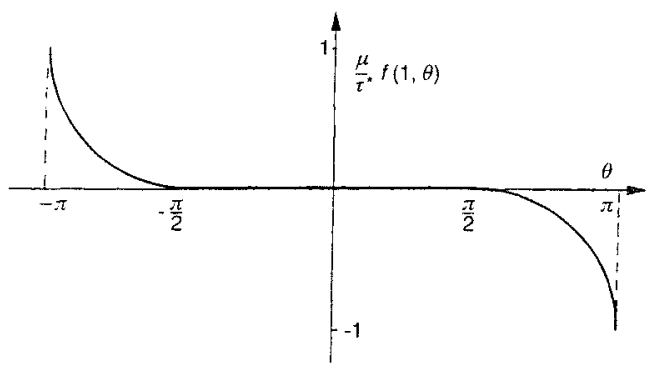

Fig. 4. Relation ship between dimensionless function $f(1, \theta)$ with $\theta$ $\left\{\begin{array}{l}\xi^{2}\left(1-\xi^{2}\right) \frac{\mathrm{d}^{2} u}{\mathrm{~d} \xi^{2}}+\xi\left(3-2 \xi^{2}\right) \frac{\mathrm{d} u}{\mathrm{~d} \zeta}+\left(1-m^{2}\right) u=0 \\ \frac{\mathrm{d}^{2} v}{\mathrm{~d} \vartheta^{2}}+m^{2} v=0\end{array}\right.$

where the eigenvalue $m$ is to be determined by the boundary conditions. The general solution of second equation of $(50)$ is

$v(\theta)=C_{1} \cos m \theta+C_{2} \sin m \theta$.

From the second equation of (43) we know that $w=0$ for $\theta=0$, then $v(0)=0$ gives $C_{1} \equiv 0$, from (51) we obtain

$v(\theta)=C_{2} \sin m \theta$.

From (44), (49) and (51) we have the displacement in the $z$-direction as follows

$w(r, \theta, t)=r u\left(\frac{r}{c_{s} t}\right) \sin m \theta, \quad r \in\left(0, c_{s} t\right)$.

The stress distribution is

$\sigma_{z y}=\mu \frac{\partial w}{\partial y}=\mu\left(\frac{\partial w}{\partial r} \sin \theta+\frac{1}{r} \frac{\partial w}{\partial \theta} \cos \theta\right)$,

i.e.,

$\sigma_{z y}=\mu\left[\left(u+r \frac{\partial u}{\partial r}\right) \sin m \theta \sin \theta+u m \cos m \theta \cos \theta\right]$.

The restriction for the cylindrical wave on the surface of the crack is

$\sigma_{z y}(r, \pi, t)=0$,

we have from (54)

$\cos m \pi=0$,

from which the eigenvalue is determined to be

$m=\frac{2 n+1}{2} \quad(n=0,1,2,3 \ldots)$.

The first equation in (50) is an ordinary differential equation with three singular points, these three singular points are

$\check{\zeta}_{1}=0, \quad \xi_{2}=-1, \quad \xi_{3}=1$,

which are all regular singular points (appendix A), thus this equation is called Riemann's equation [27], it is also called Papperitz equation. The general form of Riemann's equation is [27]

$$
\begin{aligned}
\frac{\mathrm{d}^{2} u}{\mathrm{~d} z^{2}} & +\left(\sum_{n=1}^{3} \frac{1-x_{n}-x_{n}^{\prime}}{z-z_{n}}\right) \frac{\mathrm{d} u}{\mathrm{~d} z} \\
& +\left[\sum_{n=1}^{3} \frac{\alpha_{n} \alpha_{n}^{\prime}\left(z_{n}-z_{n+1}\right)\left(z_{n}-z_{n+2}\right)}{z-z_{n}}\right] \\
& \times \frac{u}{\left(z-z_{1}\right)\left(z-z_{2}\right)\left(z-z_{3}\right)}=0,
\end{aligned}
$$


where $z=z_{n}(=1,2,3)$ are the three singular points, $x_{n}$ and $\alpha_{n}^{\prime}$ are the exponents belonging to $z=z_{n}$, and it should be noted $z_{4}=z_{1}$ and $z_{5}=z_{2}$ are used in (59). The exponents satisfy

$\sum_{n=1}^{n}\left(\alpha_{n}+\alpha_{n}^{\prime}\right)=1$.

The constants $\lambda_{n}=\alpha_{n}-\alpha_{n}^{\prime}$ are called the exponent differences. If none of them is an integer, (59) has two linearly independent solutions, if one or several of the exponent differences are integers, then the solution may involve logarithmic terms. Riemann's equation can be reduced to the Gauss' hypergeometric equation [27]

$z(1-z) \frac{\mathrm{d}^{2} u}{\mathrm{~d} z^{2}}+[c-(a+b+1) z] \frac{\mathrm{d} u}{\mathrm{~d} z}-a b u=0$,

where $a, b$ and $c$ are independent of $z$, and they are called the parameters of the equation. The three singularities of the hypergeometric equation are $0, \infty$, and 1 , they are all regular singular points.

By the symbol

$P\left\{\begin{array}{cccc}z_{1} & z_{2} & z_{3} & \\ \alpha_{1} & \alpha_{2} & \alpha_{3} & z \\ \alpha_{1}^{\prime} & \alpha_{2}^{\prime} & \alpha_{3}^{\prime} & \end{array}\right\}$,

we shall denote the complete set of solutions of (59). By noticing (A1), we have the relations for the first equation in (50) as follows

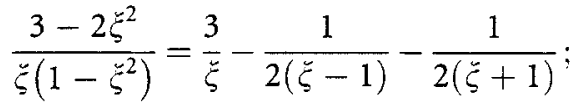

$$
\begin{aligned}
& \frac{1-m^{2}}{\zeta^{2}\left(1-\zeta^{2}\right)}=-\frac{1}{\xi(\zeta-1)(\xi+1)} \frac{1-m^{2}}{\xi},
\end{aligned}
$$

then from (59) we have the equations

$$
\left\{\begin{array}{l}
1-\alpha_{1}-\alpha_{1}^{\prime}=3 \\
1-\alpha_{2}-\alpha_{2}^{\prime}=-\frac{1}{2} \\
1-\alpha_{3}-\alpha_{3}^{\prime}=-\frac{1}{2} \\
\alpha_{1} \alpha_{1}^{\prime}=1-m^{2} \\
\alpha_{2} \alpha_{2}^{\prime}=0 \\
\alpha_{3} \alpha_{3}^{\prime}=0
\end{array}\right.
$$

the solutions to (64) are

$\alpha_{1}=-1+m, \quad \alpha_{1}^{\prime}=-1-m, \quad \alpha_{2}=0$,

$\alpha_{2}^{\prime}=\frac{3}{2}, \quad \alpha_{3}=0, \quad \alpha_{3}^{\prime}=\frac{3}{2}$.

It is easy to prove that (65) satisfies (60). The symbol expressed as (62) for the first equation in (50) is given by $P\left\{\begin{array}{cccc}0 & -1 & 1 & \\ -1+m & 0 & 0 & \zeta \\ -1-m & \frac{3}{2} & \frac{3}{2} & \end{array}\right\}$.

From (57) and (65) we have the three exponent differences as $i_{1}=2 n+1, \quad i_{2}=i_{3}=-\frac{3}{2}$.

since the first exponent difference is an integer, then we know that logarithmic terms are involved in the solutions.

The complete set of solutions of hypergeometric equation (61) are given by the symbol

$$
P\left\{\begin{array}{ccc}
0 & \infty & 1 \\
0 & a & 0 \\
1-c & b & c-a-b
\end{array}\right\} .
$$

Riemann's equation (59) for the present problem can be reduced to the hypergeometric equation (60) by the following transformation

$$
\begin{aligned}
& P\left\{\begin{array}{cccc}
z_{1} & z_{2} & z_{3} \\
\alpha_{1} & \alpha_{2} & \alpha_{3} & z \\
\alpha_{1}^{\prime} & \alpha_{2}^{\prime} & \alpha_{3}^{\prime}
\end{array}\right\}=\left(\frac{z-z_{1}}{z-z_{2}}\right)^{\alpha_{1}}\left(\frac{z-z_{3}}{z-z_{2}}\right)^{\alpha_{3}} \\
& P\left\{\begin{array}{cccc}
0 & \infty & 1 & \\
0 & \alpha_{1}+\alpha_{2}+\alpha_{3} & 0 & \frac{\left(z-z_{1}\right)\left(z_{3}-z_{2}\right)}{\left(z-z_{2}\right)\left(z_{3}-z_{1}\right)} \\
\alpha_{1}^{\prime}-\alpha_{1} & \alpha_{1}+\alpha_{2}^{\prime}+\alpha_{3} & \alpha_{3}^{\prime}-\alpha_{3}
\end{array}\right.
\end{aligned}
$$

from (66) we have

$$
\begin{aligned}
& P\left\{\begin{array}{cccc}
0 & -1 & 1 & \\
-1+m & 0 & 0 & \zeta \\
-1-m & \frac{3}{2} & \frac{3}{2}
\end{array}\right\} \\
& =\left(\frac{\zeta}{\zeta+1}\right)^{m-1} P\left\{\begin{array}{cccc}
0 & \infty & 1 & \\
0 & m-1 & 0 & \frac{2 \zeta}{\zeta+1} \\
-2 m & m+\frac{1}{2} & \frac{3}{2}
\end{array}\right\},
\end{aligned}
$$

letting $\zeta=\frac{2 \zeta}{\zeta+1}$, then $(70)$ is changed into

$$
\begin{aligned}
& P\left\{\begin{array}{cccc}
0 & -1 & 1 & \\
-1+m & 0 & 0 & \zeta \\
-1-m & \frac{3}{2} & \frac{3}{2} &
\end{array}\right\} \\
& =\left(\frac{\zeta}{2}\right)^{m-1} P\left\{\begin{array}{cccc}
0 & \infty & 1 & \\
0 & m-1 & 0 & \zeta \\
-2 m & m+\frac{1}{2} & \frac{3}{2}
\end{array}\right\}
\end{aligned}
$$

from (68) and (71) we obtain

$$
\begin{aligned}
& P\left\{\begin{array}{cccc}
0 & \infty & 1 & \\
0 & a & 0 & \zeta \\
1-c & b & c-a-b
\end{array}\right\} \\
& =P\left\{\begin{array}{cccc}
0 & \infty & 1 \\
0 & m-1 & 0 \\
-2 m & m+\frac{1}{2} & \frac{3}{2}
\end{array}\right\} .
\end{aligned}
$$

therefore the parameters of the hypergeometric equation are 
$a=m-1, b=m+\frac{1}{2}, c=2 m+1, c-a-b=\frac{3}{2}$.

Substituting (57) into (73) yields

$a=\frac{2 n-1}{2}, b=n+1, c=2(n+1), c-a-b=\frac{3}{2}$, pect to $a$ and $b$. Therefore we have

$a=n+1, b=\frac{2 n-1}{2}, c=2(n+1), c-a-b=\frac{3}{2}$.

Since $a$ and $c$ are integers greater than $1, b$ and $c-a-b$ are not integers (n.i.), therefore the two linearly independent solution expressed by hypergeometric series are

$$
\left(\frac{\zeta}{2}\right)^{\frac{2 n-1}{2}} u_{3}=\left(\frac{\zeta}{2}\right)^{\frac{2 n-1}{2}}(-\zeta)^{-(n+1)} F\left(n+1,-n ; \frac{5}{2} ; \zeta^{-1}\right)
$$

and

$$
\begin{aligned}
\left(\frac{\zeta}{2}\right)^{\frac{2 n-1}{2}} u_{6}= & \left(\frac{\zeta}{2}\right)^{\frac{2 n-1}{2}} \zeta^{-(2 n+1)}(1-\zeta)^{\frac{3}{2}} \\
& \times F\left(-n,-n+\frac{3}{2} ; \frac{5}{2} ; 1-\zeta\right),
\end{aligned}
$$

i.e.,

$$
\left(\frac{\zeta}{2}\right)^{\frac{2 n-1}{2}} u_{3}=\left(\frac{1}{2}\right)^{\frac{2 n-1}{2}}(-1)^{n+1} \zeta^{-\frac{3}{2}} F\left(n+1,-n ; \frac{5}{2} ; \zeta^{-1}\right)
$$

and

$$
\begin{aligned}
\left(\frac{\zeta}{2}\right)^{\frac{2 n-1}{2}} u_{6}= & \left(\frac{1}{2}\right)^{\frac{2 n-1}{2}}(\zeta)^{-n-\frac{3}{2}}(1-\zeta)^{\frac{3}{2}} \\
& \times F\left(-n,-n+\frac{3}{2} ; \frac{5}{2} ; 1-\zeta\right) .
\end{aligned}
$$

The two solutions of the first equation of (50) can be expressed by

$$
\begin{aligned}
u_{n}^{(1)}= & \left(\frac{1}{2}\right)^{\frac{3 n-1}{2}}(-1)^{n+1} \\
& \times\left(\frac{2 \zeta}{\zeta+1}\right)^{-\frac{3}{2}} F\left(n+1 .-n ; \frac{5}{2} ; \frac{\zeta+1}{2 \xi}\right),
\end{aligned}
$$

and

$$
\begin{aligned}
u_{n}^{(2)}= & \left(\frac{1}{2}\right)^{\frac{2 n-1}{2}}(-1)^{n+1}\left(\frac{\xi+1}{2 \zeta}\right)^{n+\frac{3}{2}}\left(\frac{1-\zeta}{1+\zeta}\right)^{\frac{3}{2}} \\
& \times F\left(-n,-n+\frac{3}{2} ; \frac{5}{2}: \frac{1-\xi}{1+\zeta}\right) .
\end{aligned}
$$

The solution of the nonzero displacement in the $z$-direction is

$$
\begin{aligned}
w(r, \theta, t)= & r \sum_{n=0}^{\infty}\left[A_{n} u_{n}^{(1)}\left(\frac{r}{c_{s} t}\right)\right. \\
& \left.+B_{n} u_{n}^{(2)}\left(\frac{r}{c_{s} t}\right)\right] \sin \frac{2 n+1}{2} \theta .
\end{aligned}
$$

where $A_{n}$ and $B_{n}$ are two constants to be determined by boundary conditions.

\section{2}

\section{Discussion}

For $n=0$, from equation (75) we obtain

$a=1, b=-\frac{1}{2}, c=2, c-a=1, c-a-b=\frac{3}{2}$,

then $(71)$ reduces to

$\left(\frac{\zeta}{2}\right)^{-\frac{1}{2}} P\left\{\begin{array}{cccc}0 & \infty & 1 & \\ 0 & 1 & 0 & \zeta \\ -1 & -\frac{1}{2} & \frac{3}{2} & \end{array}\right\}$.

One solution to the first equation of (50) for $n=0$ is expressed by

$\left(\frac{\zeta}{2}\right)^{-\frac{1}{2}} F\left(1,-\frac{1}{2} ; 2 ; \zeta\right)$

Considering (83), the following relations hold [25]

$\left\{\begin{array}{l}F(p, 1-q ; p+1 ; x)=p x^{-1} B_{x}(p, q) \\ B_{x}(p, q)=\int_{0}^{x} t^{p-1}(1-t)^{q-1} \mathrm{~d} t\end{array}\right.$,

where $p=1, q=\frac{3}{2}$, since

$B_{x}(p, q)=\int_{0}^{x} \sqrt{1-t} \mathrm{~d} t=\frac{2}{3}\left[1-(1-x)^{\frac{3}{2}}\right]$,

thus

$F\left(1,-\frac{1}{2} ; 2 ; \zeta\right)=\frac{21-(1-\zeta)^{\frac{3}{2}}}{\zeta}$.

The second linearly independent solution of

$\zeta(1-\zeta) \frac{\partial^{2} f}{\partial \zeta^{2}}+\left(2-\frac{3}{2} \zeta\right) \frac{\partial f}{\partial \zeta}+\frac{1}{2} f=0$

is $\Theta(\zeta) F\left(1,-\frac{1}{2} ; 2 ; \zeta\right)$, substituting it into the above equation we have

$\Theta^{\prime}(\zeta)=C \frac{\zeta^{4}}{\sqrt{1-\zeta}\left[1-(1-\zeta)^{\frac{3}{2}}\right]^{2}}$.

It is very difficult to obtain $\Theta(\zeta)$ by integrating (90). Nevertheless, when $\zeta$ is small, we know from (90) that $\Theta^{\prime}(\zeta) \sim \zeta$, therefore $\Theta(\breve{\zeta}) \sim \breve{\zeta}^{2}$. The two linearly independent solutions for $n=0$ are

$f_{0}^{(1)}=\sqrt{2} \zeta^{-\frac{3}{2}}\left[1-(1-\zeta)^{\frac{3}{2}}\right]: \quad f_{0}^{(2)}=\zeta^{\frac{1}{2}}\left[1-(1-\zeta)^{\frac{3}{2}}\right]$,

since $\zeta=\frac{2 \zeta}{1+\zeta}$. Then with $\xi=\frac{r}{c_{s} t} \rightarrow 0$ we get 
$f_{0}^{(1)} \rightarrow \sqrt{\frac{c_{s} t}{r}} ; \quad f_{0}^{(2)} \rightarrow\left(\frac{r}{c_{s} t}\right)^{\frac{3}{2}}$.

From (82) we know that the above mentioned first solution corresponds to

$w_{0}^{(1)} \sim \sqrt{c_{s} t r}$;

the corresponding stress is of the order

$\sigma_{z y}(r, 0, t) \sim \sqrt{\frac{c_{s} t}{r}}$.

The above relation is well-known for the stress having singularity of $r^{-1 / 2}$. The second solution in (92) corresponds to the stress

$\sigma_{z y}(r, 0, t) \sim\left(\frac{r}{c_{s} t}\right)^{\frac{3}{2}}$,

which does not produce stress singularity.

From appendix B we know that (82) contains the term as follows

$\frac{r}{c_{s} t}\left(\frac{1+\frac{r}{c_{s} t}}{2 \frac{r}{c_{s} t}}\right)^{\frac{3}{2}} \sim \sqrt{\frac{c_{s} t}{r}}$.

The contribution of this term to the displacement is

$\frac{w(r, \theta, t)}{c_{s} t} \sim \sqrt{\frac{c_{s} t}{r}} \sin \frac{\theta}{2}, \quad \frac{r}{c_{s} t} \rightarrow 0$.

Considering the propagation of the stress wave, the above equation can be rewritten as

$w(r, \theta, t) \sim \frac{A}{\sqrt{2 \pi r}} \sin \frac{\theta}{2} H\left(c_{s} t-r\right)$

where $A$ is a function of time. This term corresponds to the stress having singularity of $r^{-3 / 2}$. It is worthy to mention, that this term is similar to the relation suggested by Prakash et al. [28], when they studied the mechanism of dynamic initiation of a crack under short-duration pulse. Their relation is

$w(r, \theta, t) \sim \frac{2 \epsilon k_{0}}{\mu \sqrt{2 \pi r}} \sin \frac{\theta}{2} H\left(t-\frac{r}{c_{s}}\right)$,

where $\epsilon$ is the radius of a very small hole at the tip of the crack, and $k_{0}$ is the initial stress intensity factor. The traction-free hole was supposed to be suddenly formed at the crack tip, and this is considered coincidence with the sudden initiation of the crack. The corresponding particle velocity jump (the spike) is

$\dot{w} \sim\|w\| \delta\left(t-\frac{r}{\mathcal{C}_{s} t}\right), \quad\|w\|=\frac{A}{\sqrt{2 \pi r}} \sin \frac{\theta}{2}$

which means that the jump has singularity of order $r^{-1 / 2}$, the arrival time of the spike is $t=r / c_{s}$, and the familiar relation $\delta\left(t-r / c_{s}\right)=\mathrm{d} H\left(t-r / c_{s}\right) / \mathrm{d} t$ is used here.

4

\section{Conclusion}

A brief review is given in the first part of this paper on the application of similarity methods to fracture mechanics.
This includes some dimensionless numbers, scale invariance and some scaling laws in fracture mechanics, the emphasis is placed on the DSI and log-periodic corrections to the scaling exponents. The analogy between multiple fracture and fully developed turbulence, and the SOC property of the fracture process are also included in this part.

A novel method is given in the second part of this paper for the problem of semi-infinite mode III stationary crack under step dynamic loading, this problem is solved by similarity transformations, and the analytical solutions are expressed by hypergeometric functions. This method can also be used to solve the semi-infinite mode I and mode II stationary crack problems under the same step dynamic loading.

\section{Appendix A}

On the property of the singular points

of the first equation in (50)

The first equation in (50) can be rewritten as

$\frac{\mathrm{d}^{2} u}{\mathrm{~d} \xi^{2}}+\frac{3-2 \xi^{2}}{\xi\left(1-\xi^{2}\right)} \frac{\mathrm{d} u}{\mathrm{~d} \xi}+\frac{m^{2}}{\xi^{2}\left(1-\xi^{2}\right)} u=0$.

It has three singular points with $\xi=0,1,-1$. For $\xi=0$, since the two limits

$\lim _{\xi \rightarrow 0} \xi \times \frac{3-2 \xi^{2}}{\zeta\left(1-\xi^{2}\right)}=3 ; \quad \lim _{\xi \rightarrow 0} \xi^{2} \times \frac{m^{2}}{\xi^{2}\left(1-\xi^{2}\right)}=m^{2}$

exist, then the singular point $\zeta=0$ is a regular one [29].

For the singular point $\xi=1$, the two limits

$\lim _{\xi \rightarrow 1}(\zeta-1) \times \frac{3-2 \xi^{2}}{\xi\left(1-\xi^{2}\right)}=-\frac{1}{2} ;$
$\lim _{\xi \rightarrow 1}(\xi-1)^{2} \times \frac{m^{2}}{\xi^{2}\left(1-\xi^{2}\right)}=0$,

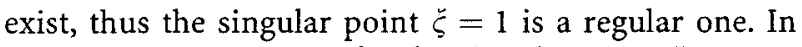
quite the same manner, for the singular point $\check{\xi}=-1$, the two limits

$\lim _{\zeta \rightarrow-1}(\xi+1) \times \frac{3-2 \xi^{2}}{\xi\left(1-\xi^{2}\right)}=-\frac{1}{2}$

$\lim _{\zeta \rightarrow-1}(\zeta+1)^{2} \times \frac{m^{2}}{\zeta^{2}\left(1-\breve{\zeta}^{2}\right)}=0$

exist, then the singular point $\xi=-1$ is also a regular one.

\section{Appendix B}

The definition of the hypergeometric series $F(a, b ; c ; z)$

The hypergeometric series $F(a, b ; c ; z)$, also called hypergeometric function, is defined by

${ }_{2} F_{1}(a, b: c ; z)=\sum_{n=0}^{\infty} \frac{(a)_{n}(b)_{n}}{n !(c)_{n}} z^{n}$.

where the subscript 2 indicates the two expressions $(a)_{n}(b)_{n}$ are in the numerator while the subscript 1 indicates the single expression $(c)_{n}$ in the denominator [29]. 
For most cases, the two subscripts can be omitted. $(a)_{n}$ is known as a Pochhammer symbol defined by

$(a)_{n}=\frac{\Gamma(a+n)}{\Gamma(a)}$.

The hypergeometric series $F(a, b ; c ; z)$ can be represented in integral form as follows

$$
F(a, b ; c ; z)=\frac{\Gamma(c)}{\Gamma(b) \Gamma(c-b)} \int_{0}^{1} \frac{t^{b-1}(1-t)^{c-b-1}}{(1-z t)^{a}} \mathrm{~d} t
$$

provided $c>b>0$. For example, the hypergeometric series in ( 80 ) for $n=1$ can be expressed by elementary function as

$$
\begin{aligned}
& F\left(2,-1 ; \frac{5}{2} ; \zeta^{-1}\right)=F\left(-1,2 ; \frac{5}{2} ; \zeta^{-1}\right) \\
& =\frac{\Gamma\left(\frac{5}{2}\right)}{\Gamma(2) \Gamma\left(\frac{1}{2}\right)} \int_{0}^{1} \frac{t\left(1-\zeta^{-1} t\right)}{\sqrt{1-t}} \mathrm{~d} t=1-\frac{4}{5} \zeta^{-1} .
\end{aligned}
$$

Similarly, the hypergeometric series in (82) for $n=1$ can be expressed by elementary function as

$$
\begin{aligned}
& F\left(-1, \frac{1}{2} ; \frac{5}{2} ; 1-\zeta\right) \\
& =\frac{\Gamma\left(\frac{5}{2}\right)}{\Gamma\left(\frac{1}{2}\right) \Gamma(2)} \int_{0}^{1} \frac{(1-t)[1-(1-\zeta) t]}{\sqrt{t}} \mathrm{~d} t \\
& =1-\frac{1}{20}(1-\zeta) .
\end{aligned}
$$

\section{References}

1. Hossdorf H (1974) Model analysis of structures. Van Nostrand Reinhold Company

2. Cherepanov GP (1979) Mechanics of brittle fracture. McGraw-Hill International Book Company

3. Bazant Z (1997) Scaling of structural failure. Appl. Mech. Rev., 50, 593-627

4. Zhao YP (1998) Suggestion of a new dimensionless number for dynamic plastic response of beams and plates. Arch. Appl. Mech., 68, 524-538

5. Zhao YP (1998) Predictions of structural dynamic plastic shear failure by Johnson's damage number. Forsch. Ingenieurwes., 63, 349-352

6. Carpinteri A (1982) Notch sensitivity in fracture testing of aggregative materials. Engineering Fracture Mechanics, 16, $467-481$

7. Zhao YP (1997) On some dimensionless numbers in fracture mechanics. Int. J. Fracture, 83, L7-L13
8. Carpinteri A (1991) Size-scale transition from ductile to brittle failure: structural response vs crack growth resistance curve. Int. J. Fracture, 51, 175-186

9. Barenblatt GI (1993) Some general aspects of fracture mechanics. Modeling of Defects and Fracture Mechanics, Herrmann G ed., Springer-Verlag

10. Barenblatt GI (1993) Micromechanics of fracture. Theoretical and Applied Mechanics 1992, Bodner SR et al. eds., Elsevier Science Publishers B.V

11. Zhao YP (1996) Irwin number and ductile-brittle fracture transition. Int. J. Fracture, 75, R17-R21

12. Mecholsky JJ, Freiman SW (1991) Relationship between fractal geometry and fractography. J. Am. Ceram. Soc., 74, 3136

13. Sornette D (1998) Discrete-scale invariance and complex dimensions. Phys. Reports, 297, 239-270

14. Brown SR, Scholz CH (1985) Broad bandwidth study of the topography of natural rock surfaces. J. Geophys. Res., 90, 12575-12592

15. Power WL, Tullis TE, Brown SR, Boitnott GN, Scholz CH (1987) Roughness of natural fault surfaces. Geophys. Res. Lett., 14, 29-32

16. Anifrani JC, Floc'h CL, Sornette D, Souillard B (1995) Universal log-periodic correction to renormalization group scaling for rupture stress prediction from acoustic emissions. J. Phys. I France, 5, 631-638

17. Sornette D, Sammis CG (1995) Complex critical exponents from renormalization group theory of earthquakes: implications for earthquake predictions. J. Phys. I France, 5, 607-619

18. Blumenfeld R, Ball RC (1991) Onset of scale-invariant patten in growth processes: the cracking problem. Physica A, 177, 407-415

19. Novikov EA (1966) Mathematical model for the intermittence of turbulent flow. Sov. Phys. Dokl., 11, 433-439

20. Frisch U (1995) Turbulence-The Legacy of A. N. Kolmogorov. Cambridge University Press

21. Zhao YP (1998) Explaining the analogy between dislocation line in crystal and vortex filament in fluid. Mech. Res. Comm. (in press)

22. Borodich FM (1997) Some fractal models of fracture. J. Mech. Phys. Solids, 45, 239-259

23. Bak P, Tang C, Wiesenfeld K (1987) Self-organized criticality: an explanation of $1 / f$ noise. Phys. Rev. Lett., 59, 381-384

24. Bak P, Tang C (1989) Earthquakes as a self-organized phenomenon. J. Geophys. Res., 94, 15635-15637

25. Ito K, Matsuzaki M (1990) Earthquakes as self-organized critical phenomena. J. Geophys. Res., 95, 6853-6860

26. Freund LB (1990) Dynamic fracture mechanics, Cambridge University Press

27. Erdelyi A, Magnus W, Oberhetting F, Tricomi F (1953) Higher transcendental functions, McGraw-Hill Book Company, Inc

28. Prakash V, Freund LB, Clifton RJ (1992) Stress wave radiation from a crack tip during dynamic initiation, J. Appl. Mech., 59, 356-365

29. Humi M, Miller W (1990) Secondary course in ordinary differential equations for scientists and engineers, SpringerVerlag 